\title{
Localization by Salp Swarm Optimization with Doppler Effect in Wireless Sensor Networks
}

\author{
${ }^{1}$ Panimalar Kathiroli \\ Research Scholar, Department of Computer Science and Engineering, Pondicherry Engineering College, Puducherry \\ E-mail: panimalar2688@pec.edu, ORCID:0000-0002-9020-9646
}

${ }^{2}$ Kanmani. S

Professor, Department of Information Technology, Pondicherry Engineering College, Puducherry, India E-mail: kanmani@pec.edu, ORCID: 0000-0001-8344-1757

Received: 01 June 2021; Accepted: 28 October 2021; Published: 08 December 2021

\begin{abstract}
Wireless sensor networks (WSNs) have lately been widely used due to its abundant practice in methods that have to be spread over a large range. In any wireless application, the position precision of node is an important core component. Node localization intends to calculate the geographical coordinates of unknown nodes by the assistance of known nodes. In a multidimensional space, node localization is well-thought-out as an optimization problem that can be solved by relying on any metaheuristic's algorithms for optimal outputs. This paper presents a new localization model using Salp Swarm optimization Algorithm with Doppler Effect (LOSSADE) that exploit the strengths of both methods. The Doppler effect iteratively considers distance between the nodes to determine the position of the nodes. The location of the salp leader and the prey will get updated using the Doppler shift. The performance validation of the presented approach simulated by MATLAB in the network environment with random node deployment. A detailed experimental analysis takes place and the results are investigated under a varying number of anchor nodes, and transmission range in the given search area. The obtained simulation results are compared over the traditional algorithm along with other the state-of-the-art methods shows that the proposed LOSSADE model depicts better localization performance in terms of robustness, accuracy in locating target node position and computation time.
\end{abstract}

Index Terms: Doppler Effect, Localization, WSN, Salp Swarm Algorithm, EHO, GWO, BSA, DV-Hop.

\section{Introduction}

Modern-day advance in micro-electromechanical systems and transmission knowledge led to change of economically, multi-operative, small smart nodes. This collection of independent nodes gives competencies to communicate with other nodes in the wireless medium known as Wireless Sensor Networks [1]. Various components of the node are sensing unit, processing unit, communication unit, memory unit, power unit and location unit [2]. The sensing unit with an external and internal sensor assists in perceiving the data from the physical world like light, pressure, humidity, tension, temperature, sound, etc. The nodes sense these data and send it to a Base Station (BS) by multi-hop communication. Analog-to-Digital Converter (ADC) converts the collected analog signals to digital. The processing unit controls other components and process the received signals. The transceiver is the most powerconsuming unit that cooperates with other nodes and the rest of the parts. The power unit supplies energy using smallsized batteries or capacitors equipped in the node. The location unit in the nodes is responsible for finding the location of the node and this unit is optional. Previously for locating nodes [3], Global Positioning System (GPS) has been fixed with nodes at the time of deployment. But it become an impractical solution due to its expense, inaccessibility nature when placed indoors, and disruption during environmental circumstances. The node localization is a multidimensional optimization problem [4] that can be solved using population-based models. The data gathered by arbitrarily placed nodes in harsh areas is not applicable when the location of nodes is unknown. Hence, the demand for accurate node localization model becomes highly essential since the nodes cannot be changed or batteries cannot be recharged manually. The number of data transferred in the network and the size of the packet sent affect the cost. During the broadcast, the packet is received to all the nodes with location information. This value is proportional to the number of neighbor nodes. So, it covers more nodes within the given communication range in the network. This makes the communication cost low in the proposed method. Still energy consumption in nodes is the challenging issue in WSN. By managing the energy used by the node increases the network lifetime. It also measures the efficiency of localization method. As of the literature, Meta-heuristics methods are the eminent category of optimization techniques that attain quality solutions for the target problem in sensible time. In the last few decades, several optimization methods are 
utilized effectively to report the issues of node localization in WSNs [5]. By no free lunch theorem [6], every approach has its own advantage and disadvantage. Many hybrid approaches have been initiated for unravelling localization problem to obtain lesser localization error. An effective bat algorithm with doppler effect for node localization [7] updates the frequency in the moving bats and converge quickly. The localization error is lessened using two parameters that balances the algorithm and normalize the performance.In each step of multi-stage localization, Elephant Herding Optimization EHO [8,9] inspired by elephants used to locate target nodes with three or more neighboring anchor nodes. Nodes which are localized in first stage become anchor nodes for the subsequent stage and the process is iterated until all target nodes are localized. Though this method provides robustness, but it lacks in providing energy while locating nodes in large scale WSN. To further improvise localization using EHO, it is been hybridized with tree growth optimization algorithm and empirical experiments are performed in diverse sizes of sensor networks to show better performance of the anticipated model. Localization model by Butterfly Optimization Algorithm (BOA) [10] computes distance along with Gaussian noise. The localization accuracy result is optimal but still localization rate with minimal error can be enhanced. For UWSN [11], a doppler shift-based localization method with genetic algorithm improves accuracy in localizing target nodes. In marine environment, when source and observer are mobile this algorithm depends on Doppler shift of sound waves. The doppler shift regulates the estimated location of target node and GA restricts localization error. As underwater nodes are reduced by water currents, the shift in frequency aids in improved location estimation of a target node within a given area. In irregular fields the node that can get data from anchor nodes need not be convinced. Hence, estimating the position of a node as centroid results in high location estimation errors. The performance even gets worser in case of nodes on the borders of network holes and fields. If there are obstacles and holes, then the nodes cannot be uniformly distributed. For the anisotropic environment, a Mamdani fuzzy model is employed for modeling the edge weights between target and anchor nodes. Weighted centroid method is used to estimate the target node location. In absolute positioning scheme [12], a localized Autonomous Underwater Vehicle (AUV) is built on time difference between the signal arrivals and depth information received from an on-board pressure sensor. A cross-examination pulse is conveyed at a fixed rate by AUV and is acknowledged at the surface laterally with response from individual transponder. In UASNs, the main constraints are clock synchronization and mobility. The hybrid architecture [13] is put forth for the AUV active and passive nodes. The auxiliary information of nodes is obtained by AUVs. The localization process is initialized by active sensor nodes broadcast messages that are received by both AUVs and the passive sensor nodes. The passive nodes do not broadcast and localize themselves by snooping the messages sent by the anchor nodes. An algorithm [14] is instigated to find the position of the nodes which is then improved by using an iterative least square method. In [15] Particle Swarm Optimization (PSO) algorithm uses the velocity of moving particles locating the position of target nodes to minimize localization errors. Further niching method with PSO is used to solve ambiguity issue resulting in collinear anchors. Tree criteria are also provided for selecting reliable neighboring anchors while the unknown node is localized. Also, PSO with variable neighborhood search (HPSOVNS) in an outdoor atmosphere uses mean squared range error and Received Signal Strength Indicator for locating adjacent anchor nodes and to find the distances among nodes. Enhanced PSO algorithm for node segmentation [16], separate nodes into segments to improve the precision of the estimated distances between nodes. From the above-mentioned existing algorithms, the use of anchor nodes locates the unknown nodes. If the distance estimated from the anchor to target node is more precise, then the localization accuracy of the target node can be improved. So, if some anchor nodes have high estimated distance error then they are discarded and only part of them are applied for positioning, the localization precision can be enhanced. The main contributions in this paper are briefed as follows:

1. The target node location is calculated by the trilateration method with help of salp swarm optimization algorithm fitness function. The Doppler effect method helps to determine the position of the nodes iteratively by considering Euclidian distance. The location of the salp leader will update using the Doppler frequency.

2. To decrease the energy consumption and to maximize the network lifetime, we present an effective node localization algorithm using of Salp Swarm Optimization Algorithm with Doppler Effect (LOSSADE). The combination of salp swarm behavior and DE takes place to effectively localize the position of the unknown nodes using anchor nodes.

3. LOSSADE performance validation is investigated using parameters like localization error, discovered nodes, localization time, transmission range, anchor nodes.

The remaining of this paper is organized as follows. Section 2 gives the introduction on salp swarm algorithm, Section 3 presents the implementation background of localization model using the enhanced salp swarm optimization model with doppler effect and Section 4. presents the experimental setup and performance metrics of localization; Section 5 gives the simulation results and discussion. Finally, Section 6 summarized Conclusions and suggestions for future works. 


\section{Related Work}

\subsection{Salp Swarm Optimization Algorithm}

Salp swarm algorithm (SSA) [17] is a metaheuristic algorithm inspired from foraging behavior of complex organism called salps belonging to the family of Salpidae in deep oceans by S.Mirjalili, Amir H.Gandomi, Shahzrad Saremi, Hossam Faris, Seyedeh Zahra Mirjalili, M. Mirjalili in 2017. Salp is a which comes under the classification of chordates. These have a barrel-shaped structure depicted in Fig. 1(a) and transparent body. The salp has tails, gills, a primitive eye and enlarged brain. It lives on plankton and organic matter. A biological study is defined as primary milestones due to the complexity in the living environment and no access is provided, especially it is tedious to manage in laboratories. In deep oceans, Salps swarm constitute a chain akin to structure known as salp chain as depicted in Fig. 1(b). They move forward in the water by pumping through body as propulsion. A major cause for these behaviors is undesirable; however, the developers hoped that it is carried out for accomplishing the best locomotion with the help of fast coordinated modifications as well as foraging. Individual salp moves by contracting its body in water. When the water is pumped through the body as propulsion the salp moves towards and expels out. Microscopic plants, phytoplankton are filtered by internal feeding filters. The prey gets trapped inside and digestion takes place.

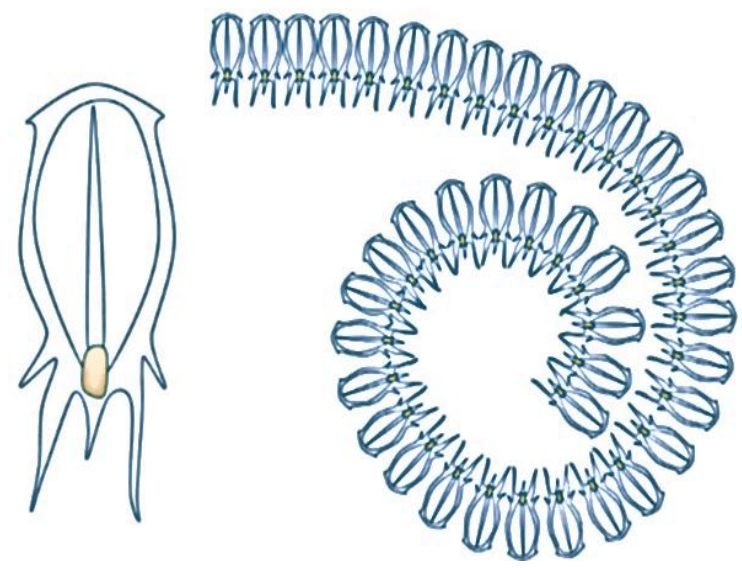

Fig.1. (a) Individual salp; (b) swarm of salps

To mathematically model the salp chains initially, the population is first divided into two groups: leader and followers. The salp at the foreside of the chain link is called a leader, whereas the rest are called followers. The leader guides the swarm and the follower follows the leader. The SSA flowchart is given in figure 2. In a d-dimensional space, the current position of leader $p_{d}^{L}$ is updated for the food source $F_{d}$ and is delineated by the equation (1), where UB $\mathrm{B}_{\mathrm{d}}$ and $\mathrm{LB}_{\mathrm{d}}$ are the upper and lower bound respectively to $\mathrm{d}^{\text {th }}$ dimension. Coefficient $r_{1}, r_{2}$ balances between exploration and exploitation, $r_{3} \in[0,1]$ decides the next position of the leader.

$$
p_{d}^{L}=\left\{\begin{array}{l}
F_{d}+r_{1}\left(\left(U B_{d}-L B_{d}\right) r_{2}+L B_{d}\right) r_{3} \geq 0 \\
F_{d}-r_{1}\left(\left(U B_{d}-L B_{d}\right) r_{2}+L B_{d}\right) r_{3}<0
\end{array}\right.
$$

Here $r_{1}$ decreases over iteration. $r_{1}=2 e^{\left(-\frac{4 I^{(t)}}{I_{\max }}\right)^{2}}$, where $\mathrm{I}^{(\mathrm{t})}$ is current iteration and $\mathrm{I}_{\max }$ is the maximum number of iterations. To update the position of followers, by Newton's law of motion the equation (2) displays the $i^{\text {th }}$ salp's followers' position in $\mathrm{d}^{\text {th }}$ dimension. $v_{0}$ represents the initial speed, 't' denotes a time, $\alpha=\frac{v_{N}}{v_{0}}$, where, $v=\frac{p-p_{0}}{t}$. The time in optimization discrepancy between iterations is equal to 1 .

$$
p_{d}^{i}=\frac{1}{2} \alpha t^{2}+v_{0} t
$$

Considering $v_{0}=0$, where $\mathrm{i} \geq 2, p_{d}^{i}$ is $\mathrm{i}^{\text {th }}$ followers' position in $\mathrm{d}^{\text {th }}$ dimension and the salp chain is given in equation (3).

$$
p_{d}^{i}=\frac{1}{2}\left(p_{d}^{i}+p_{d}^{i-1}\right)(\text { Where } \mathrm{i} \geq 2)
$$




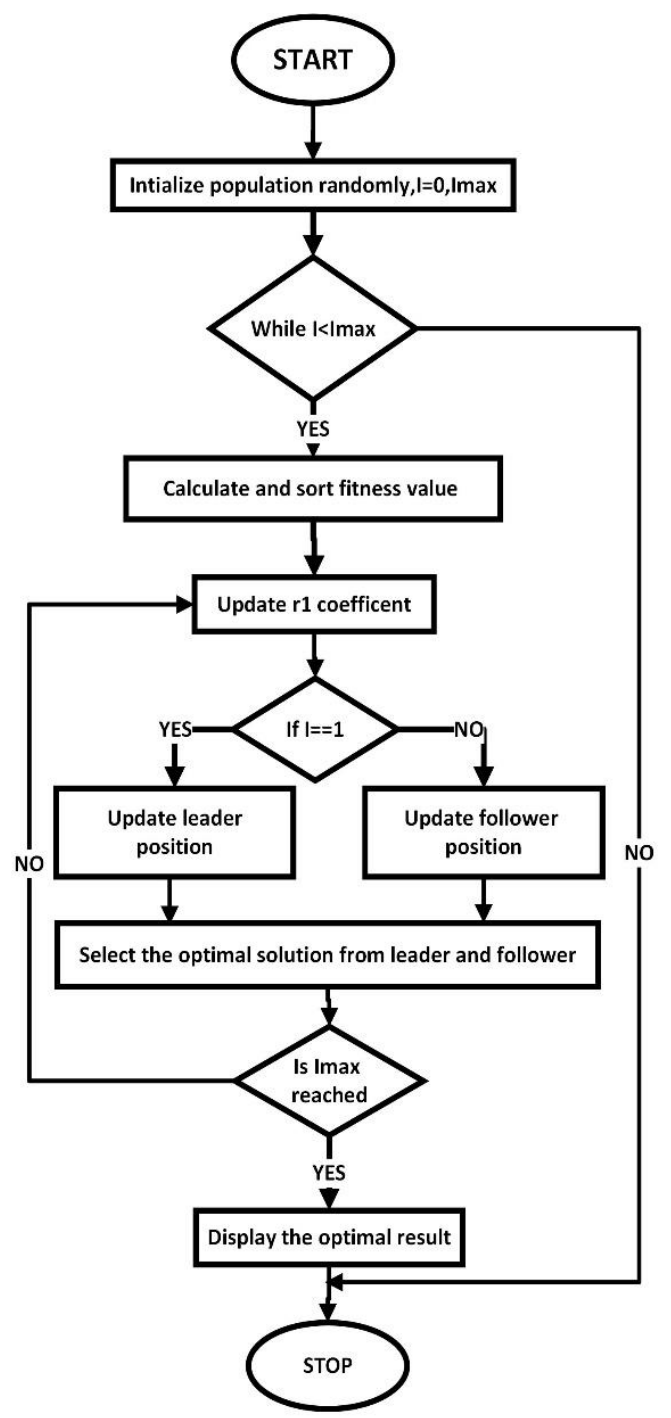

Fig.2. Flowchart of Salp Swarm optimization algorithm

\section{Research Methodology}

A WSN is a grid with a cluster of 'TN' target nodes (unknown nodes) and 'AN' anchor nodes (known or beacon nodes). The nodes without localization information are randomly scattered in the target region. The AN's key role is to send anchor signals and messages to allow TN to discover themselves. Each unknown node receives the anchor signals at a fixed time and cites the related data [2]. For the given two-dimensional range, to build a network the nodes are randomly organized in a target region. These nodes are incapable of relocating when they are positioned. The organized network has a sink node placed at a stationary location. The network contains AN distributed randomly with known position either through GPS or manually, and TN. The deployed nodes can communicate with each other if the distance between them is less than the transmission range $T_{R}$. Different ranging methods can be used to estimate the distances amid nodes. The given strategy has been employed for localizing the nodes in WSN.

1. Initialize $A N$ with coordinates $\left(\mathrm{x}_{\mathrm{i}}, \mathrm{y}_{\mathrm{i}}\right)$ where $\mathrm{x}_{\mathrm{i}}=\left[\mathrm{x}_{1}, \mathrm{x}_{2} \ldots \mathrm{x}_{\mathrm{AN}}\right], \mathrm{y}_{\mathrm{i}}=\left[\mathrm{y}_{1}, \mathrm{y}_{2} \ldots \mathrm{y}_{\mathrm{AN}}\right]$ and TN with coordinates $(\mathrm{x}, \mathrm{y})$. Each AN knows its location and TN need to estimate the location using these neighboring AN. The distributed range-based localization technique (like signal propagation time or received signal strength indicator) is used to estimate the coordinates of sensor nodes. Every AN and TN have $T_{R}$.

2. Euclidean distance between each $\mathrm{TN}$ and $\mathrm{AN}$ is calculated and altered by Additive Gaussian White Noise. Each TN calculates the distance $\widehat{\mathrm{d}}_{\mathrm{i}}$ to an AN using equation (4), $\mathrm{d}_{\mathrm{i}}$ is the actual distance calculated using equation (5).

3. It is the distance between $\mathrm{AN}\left(\mathrm{x}_{\mathrm{i}}, \mathrm{y}_{\mathrm{i}}\right)$ and $\mathrm{TN}\left(\mathrm{x}_{\mathrm{j}}, \mathrm{y}_{\mathrm{j}}\right)$. The uniformly distributed random valued $\mathrm{n}_{\mathrm{i}}$ is the noise altering the measured distance in the range $d_{i} \pm d_{i}\left(\frac{P_{n}}{100}\right)$ and $P_{n}$ is the percentage of noise in the measured distance. The localization algorithm is a vital part that elucidates the use of existing data from estimated 
positions of a maximum number of sensor nodes in the target network with high accuracy.

$$
\begin{gathered}
\hat{\mathrm{d}}_{\mathrm{i}}=\mathrm{d}_{\mathrm{i}}+\mathrm{n}_{\mathrm{i}} \\
d_{i}=\sqrt{\left(x-x_{i}\right)^{2}+\left(y-y_{i}\right)^{2}}
\end{gathered}
$$

4. If there are at least three $A N$ within $T_{R}$ of $T N$. By trilateral positioning, the distance $d_{i}$ between the $A N$ $\left(\mathrm{x}_{1}, \mathrm{y}_{1}\right),\left(\mathrm{x}_{2}, \mathrm{y}_{2}\right),\left(\mathrm{x}_{3}, \mathrm{y}_{3}\right)$ to TN $(\mathrm{x}, \mathrm{y})$ is calculated using equation $(6)$. The desired node is named as localizable node when it has 3 AN inside the communication radius of the TN. Followed by the trigonometric laws of sine or cosine, the coordinates of TN are determined. In the same way, in multilateration target node estimated model, distance metrics of massive anchor nodes are applied for reducing the error from an original distance as well as evaluated distance.

$$
\left(x_{c}, y_{c}\right)=\left(\frac{1}{N} \sum_{i=1}^{N} x_{i}, \frac{1}{N} \sum_{i=1}^{N} y_{i}\right)
$$

5. In case of a localizable node, the proposed model is executed autonomously for identifying the location of the target node as in equation (7), where $\mathrm{N}$ is the overall count of anchor nodes inside the communication range of the localizable target node.

$$
f(x, y)=\frac{1}{N}\left(\sum_{i=1}^{N} \sqrt{\left(x-x_{\mathrm{i}}\right)^{2}+\left(y-y_{i}\right)^{2}}-\hat{d}\right)^{2}
$$

6. Localization Error (LE) is calculated after the estimation of all localizable TN i.e., $\mathrm{N}_{\mathrm{TN}}$ in the network. As in equation (8), $\left(\mathrm{X}_{\mathrm{i}}, \mathrm{Y}_{\mathrm{i}}\right)$ is the coordinate of the $\mathrm{i}^{\text {th }} \mathrm{TN}$ after localization and $\left(\mathrm{x}_{\mathrm{j}}, \mathrm{y}_{\mathrm{j}}\right)$ is actual coordinates of the node.

$$
L E=\frac{1}{N_{T N}} \sum_{i=1}^{N} \sqrt{\left(x_{i}-X_{\mathrm{i}}\right)^{2}+\left(y_{\mathrm{i}}-Y_{i}\right)^{2}}
$$

7. The procedure of ii to vi is repeated still the $\mathrm{TN}$ are localized. The localization model depends upon maximum localization error and count of unlocalized nodes $\mathrm{N}_{\mathrm{N}_{\mathrm{L}}}$ that can be determined under the application. The minimum scores of $\mathrm{LE}$ and $\mathrm{N}_{\mathrm{N}_{\mathrm{L}}}$ develops an effective localization as in equation (9).

$$
N_{N_{T N}}=N-N_{T N}
$$

8. The network average hop distance considers the outcome of all the nodes deployed in the given area as in equation (10). The DV Hop algorithm is a distributed range-free localization algorithm that relies on the distance vector routing protocol and network connectivity information.

$$
\text { Network Avg. Hop distance }=\frac{\sum_{i=1}^{n} \text { Avg.hop distance }}{n}
$$

The localized node count gets enhanced as the iteration develops. Also, it maximizes the anchor nodes count inside the communication radius of the localizable $\mathrm{TN}$ as the evaluated location of the TN acts as an $\mathrm{AN}$ in the consecutive iteration. It is used for limiting the issue of flip ambiguity that generates maximum LE. Thus, the processing duration for localization data of the TN enhances when the iteration is improved. The main principle within is to estimate the distances between target nodes and anchor nodes by multiplying the average hop distance in WSNs by the count of hops. This makes the communication cost problem to be highlighted.

\section{The Proposed Model- Localization using a Hybrid Salp Swarm Optimization Algorithm with Doppler Effect}

Doppler Effect (DE) or Doppler Shift (DS)[41] was formulated based on altering frequency of actual event i.e., the change in the frequency of the wave in relation to an observer who is moving towards its source. It was anticipated by an Austrian physicist Hans Christopher Doppler. Hypothetically, the DE is the change in wavelength of a source with respect to the relative movement between a receiver and the source. The salp periodically moves its body producing the current waves that travel out words in all direction at same speed since it is in the same water medium [19]. Once the waves leave the salp leader, it is no longer affected by any further movement of the observer. Hence the wave travels on 
its own. The next wave's Centre is shifted towards the prey in the direction of moving. As in figure 3, it creates the concentric circles, this reaches the prey at the same frequency. In water, the speed of the wave is calculated using the frequency of the individual salp as in the equation (11).

$$
\operatorname{Speed}(\mathrm{v})=\text { wavelength }(\lambda) \times \operatorname{Frequency}(\gamma)
$$

Using the primitive eye, salp reaches the food as a chain. The leader guides followers for moving towards the food source. The leader moves randomly and maximizes the frequency. Hence, leader (observer) can determine the distance and location of the source. The prey (source) and the individual follower salp are the observers that experiences disturbance in frequency. If the leader moves towards the prey, then each consecutive wave that is originated moves closer to the source and far from the observer. Subsequently each consecutive wave has a shorter distance to travel before reaching source and it also takes less time to reach observer. Also, if the follower moves towards the leader help to form a chain like structure each wave produced by the moving follower towards a leader has further distance to travel. The observer observes the frequency of arrival that is less than the frequency of actual wave. When the leader moves towards the prey then the velocity of the wave is positive or greater than zero [15]. The frequency of the leader detects the speed of the prey. The faster the movement of salp, the closer together the waves become. The observed frequency $(\gamma)$ is calculated by the equation (12),

$$
\gamma^{\prime}=\gamma_{0}\left[\frac{v_{0}+v_{o b s e r v e r}}{v_{0}}\right]
$$

If the leader moves away from the prey, then frequency goes down and the velocity becomes negative or less than zero. The merits of the Doppler shift are used for leader's movement as in equation (13),

$$
\gamma^{\prime}=\gamma_{0}\left[\frac{v_{0}-v_{o b s e r v e r}}{v_{0}}\right]
$$

Here $\boldsymbol{v}_{\mathbf{0}}$ is the speed of the wave, $\boldsymbol{v}_{\text {observer }}$ is the speed of the wave and $\boldsymbol{\gamma}_{\mathbf{0}}$ is the frequency. From equation. (12) and (13), equation (14) is derived

$$
\gamma^{\prime}=\gamma_{0}\left[\frac{v_{0} \pm v_{o b s e r v e r}}{v_{0}}\right]
$$

If the prey (source) moves towards the observer. It is given in equation (15),

$$
\gamma^{\prime}=\gamma_{0}\left[\frac{v_{0}}{v_{0}-v_{\text {source }}}\right]
$$

If the source moves away from the observer as in equation (16),

$$
\dot{\gamma}=\gamma_{0}\left[\frac{v_{0}}{v_{0}+v_{\text {source }}}\right]
$$
(17).

The +/- symbols are inverted as the sign on top to the relative motion of the source to the receiver as in equation

$$
\gamma=\gamma_{0}\left[\frac{v_{0}}{v_{0} \mp v_{\text {source }}}\right]
$$

Here $\boldsymbol{v}_{\text {source }}$ is the speed of the source. In the scenario of both moving observer and moving source, the frequency is high. Vice versa, if moving away then the frequency goes down [13].

$$
\gamma_{\text {observer }}=\gamma_{\text {source }}\left[\frac{v_{0} \pm v_{\text {observer }}}{v_{0} \mp v_{\text {source }}}\right]
$$

In equation (18), the wave is disturbed by the observer and reciprocated by the prey in the water medium and so the Doppler shift increases the frequency. When the prey receives the wave frequency of arrival then it reciprocates with another Doppler shift. Therefore, two doppler shift takes place which has been applied for estimating the location of the salp leader in the chain. The new frequency function is given as in equation (19),

$$
\gamma_{i}^{t+1}=\gamma_{i}^{t}\left[\frac{v_{0} \pm \hat{v}}{v_{0} \mp \dot{v}}\right]
$$


Where $\dot{v}=\frac{\boldsymbol{p}-\boldsymbol{p}_{\mathbf{0}}}{\boldsymbol{t}}$, here Salp Adjustment Operator (SAO) $r_{4} \in[0.4,1]$ of a random uniform, value is applied for enhancing the function and for the periodical outcome. This variable is responsible to balance the relationship between global solutions and local solutions. The new position of the salp for the doppler shift is given as in equation (20),

$$
p_{i}^{(\mathrm{t}+1)}=r_{4} * \frac{1}{2}\left(p_{i}^{(\mathrm{t})}+p_{i-1}^{(t)}\right) \times \gamma_{i}^{t+1}
$$

If the new position is negative, then exchange with a newly generated random number $=0$. If the new position is larger, then replace the position with the maximum value. The position is updated iteratively till the termination condition. The LOSSADE model is applied for estimating the coordinates of sensor nodes. The major goal of node localization is to compute the coordinates of desired nodes by reducing the objective function. A localization problem of WSN is assumed as an optimization issue that has been evolved by different meta-heuristic approaches. The working of the presented LOSSADE algorithm incorporates the improved Salp Swarm Optimization algorithm with the Doppler shift. Salps are invoked using the centroid of anchor nodes inside a communication radius. The optimal measure ( $\mathrm{x}, \mathrm{y}$ ) has been determined using the LOSSADE model once the number of rounds is limited. The LOSSADE approach is applicable to identify the coordinates $(\mathrm{x}, \mathrm{y})$ of $\mathrm{TN}$ which reduces the localization error. The primitives applied in the localization problem is a mean square distance among a target node as well as an $\mathrm{N}$ that has been reduced with the application, where $\mathrm{N} \geq 3$ defines the count of $\mathrm{AN}$ inside a communication radius of TN.

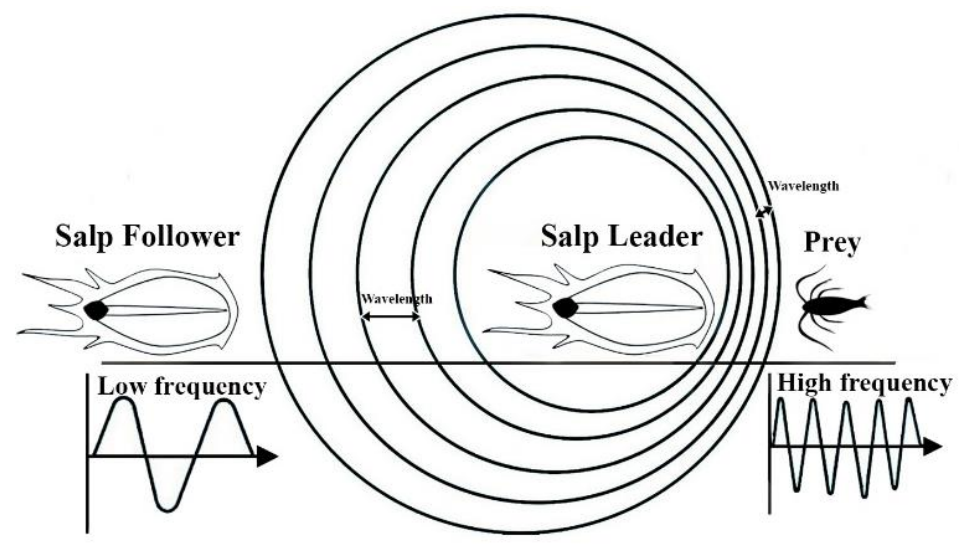

Fig.3. Enhanced Salp swarm optimization using Doppler effect

Algorithm 1: Pseudocode of hybrid Salp Swarm Optimization Algorithm with Doppler Effect

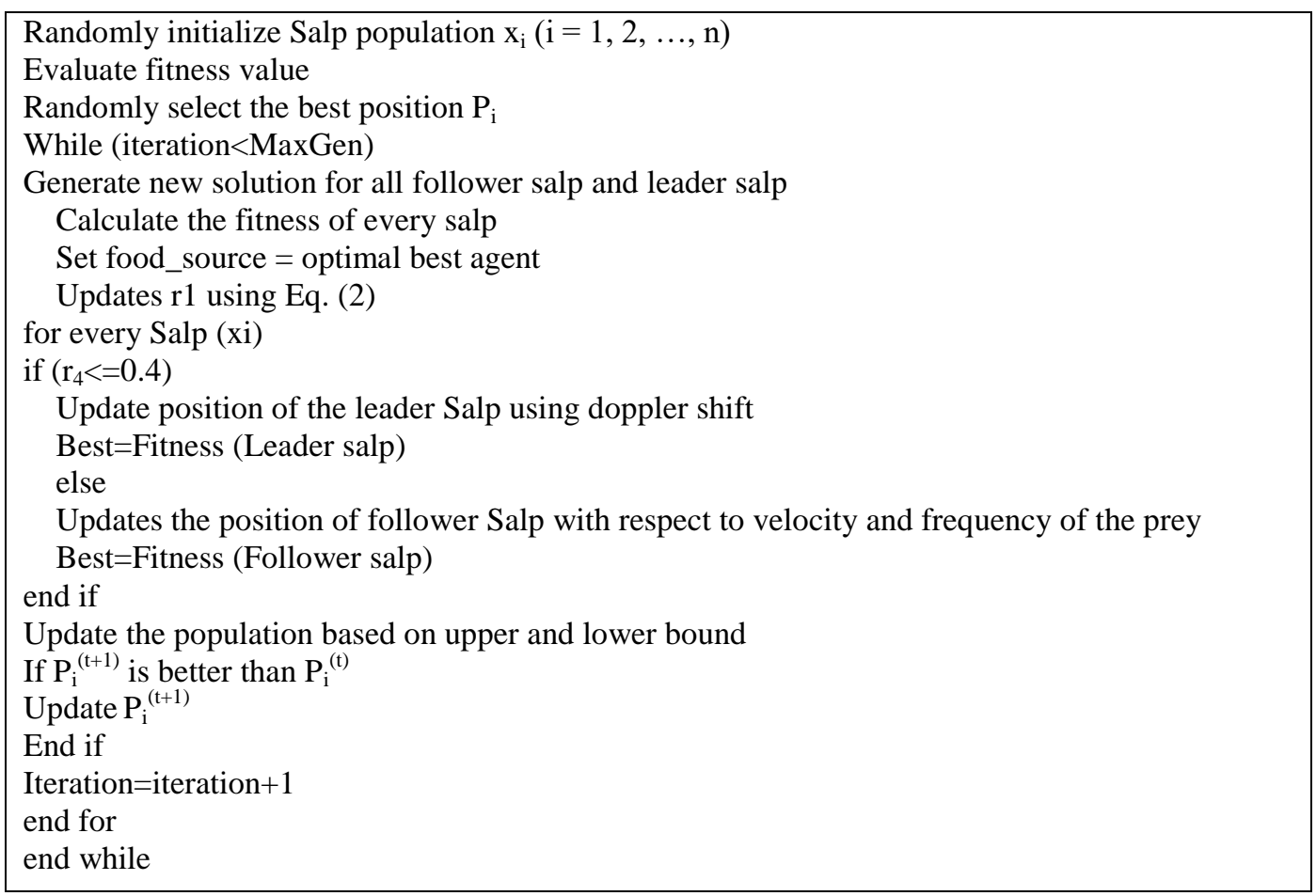




\section{Experimental and Performance Evaluation Setup}

The simulation testbed is a standard configuration of the Windows 10 (64-bits) operating system on an Intel(R) Core (TM) i5-6402P $2.80 \mathrm{GHz}$ CPU, with 16GB DDR4 memory and a 2TB hard disk. For assessment purposes, the simulation model is based on MATLAB 2018a with the standard library, which provides the simulator to implement network setups and determine localization results. Our experimental area's size is $100 \times 100 \mathrm{~m}^{2}$, in which all anchor nodes and target nodes are randomly positioned with initial energy ranging between 2-10 Joules. The number of target nodes varies from 10 to 150 to test whether the node density can affect the accuracy. We also study to what extent the number of anchor nodes varying from 10 to 50 (with a step size of 10 nodes) affects the accuracy for maximum iteration of 100. In a localization process, maximum number of iterations required relies on the energy consumption involved in refining the localization results to obtain accuracy. As energy, the primary property, it is required to be considered for computation and communication costs of the localization process. For simulation purpose, the compared state of the art algorithm used are also solved for the localization problem and the number of iterations is set to 100. During the output, the efficiency of the proposed algorithm is estimated based on several metrics, such as localization error, convergence rate, localization rate, and localization time. The node localization of the LOSSADE model shows a better performance for the deployed nodes as in the figure 4. It uses the basic SSA with the parameter fine-tuned and the salp adjustment operator $r_{4}$ with value 0.4 is considered. To find the location of 170 target node using 30 anchor nodes in the given search space indicates that the model proposed is accurate. The higher the known node density results in higher neighbor nodes to find the location of unknow nodes in the network area.

For the Salp swarm optimization-based node localization is shown in figure 5 has considered coefficient $r_{1}$ balances between exploration and exploitation, Coefficient $r_{2}$, and $r_{3}$, are given values of 0.7 and 0.3 respectively.

The Grey Wolf Optimization-based node localization as in figure 6 has considered controlling parameter which linearly decreases from 2 to 0 for exploration and exploitation, and numbers of wolf's ' $n$ ' is 200 helps to determine the localization information of target node by updating their position.

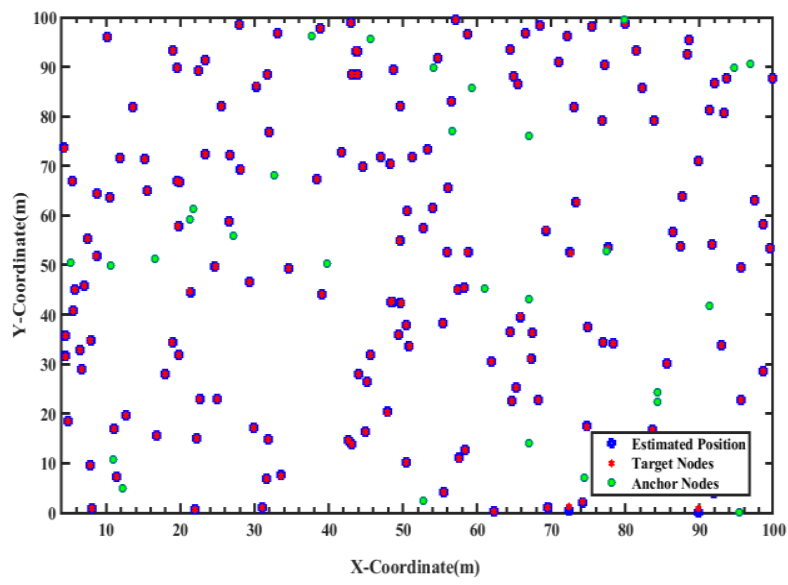

Fig.4. Localization of nodes using LOSSADE

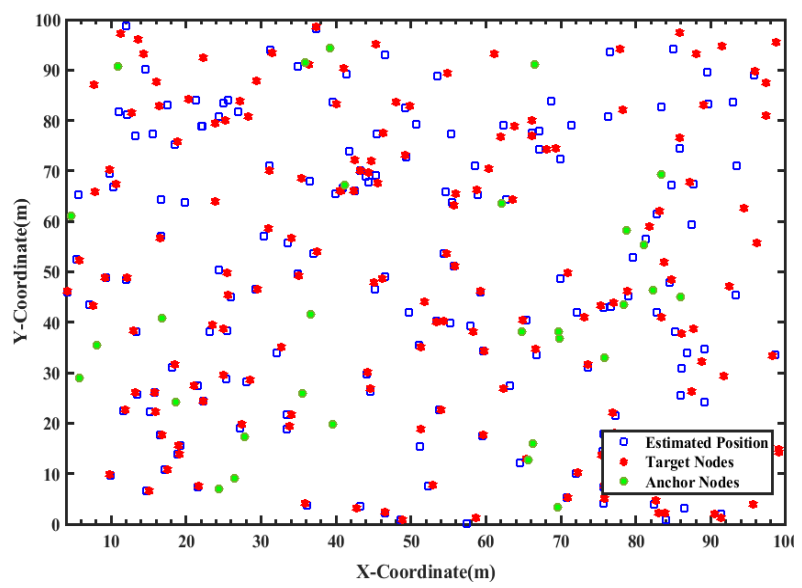

Fig.5. Localization of nodes using SSA

The Bat Search Algorithm based node localization has considered ' $\mathrm{n}$ ' as 200 bats fly randomly with varying frequency $f_{\min }=0.01 \mathrm{kHz}, \mathrm{f}_{\max }=0.05 \mathrm{kHz}$. The localization of nodes using BSA in the search area is given in figure 7 . 
The loudness 'Ai' varies from maximum value $A_{\max }=0.2 \mathrm{~ms}$ to minimum constant value $\mathrm{A}_{\min }=0$ to search prey, it varies depending on the size of the problem. The adjustment rate of emitted pulse ' $r_{i}$ ' ranges in the interval $[0,1]$ reliant on the proximity of the target prey and given as $0.5 \mathrm{~ms}$.

The Elephant Herd Optimization based node localization as in figure 8 has considered ' $n$ ' as 200 elephants, $\alpha=0.5$ is a scale operator which defines the influence of matriarch, $\beta=0.1$ is a influence operator for number of clans $=5[47]$. Figure 9 shows the DV-Hop based localization for the given AN to find TN location.

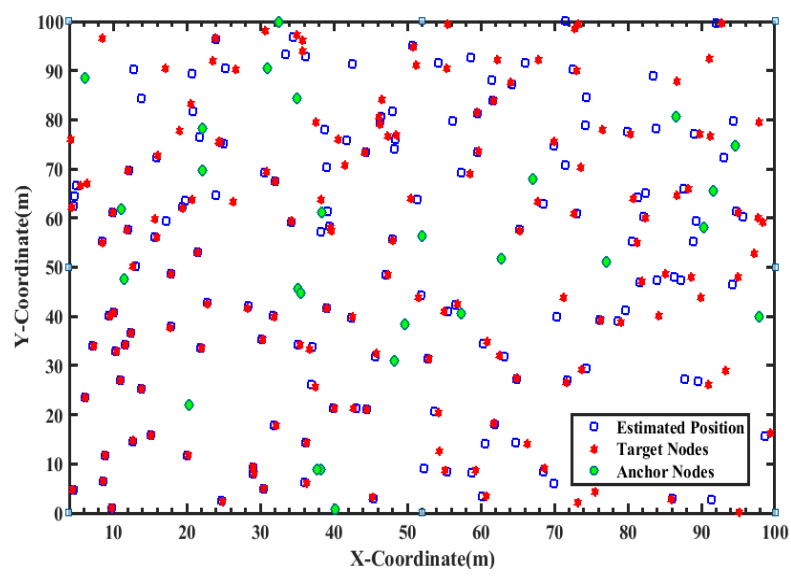

Fig.6. Localization of nodes using GWO

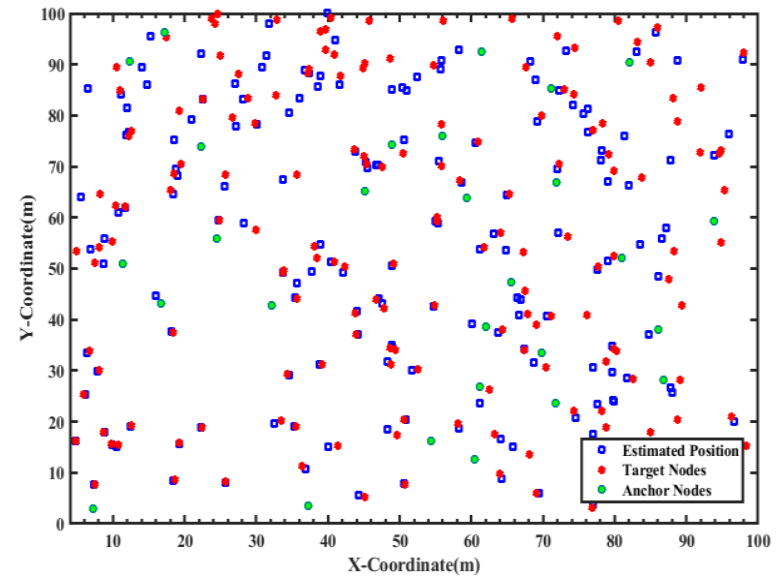

Fig.7. Localization of nodes using BSA

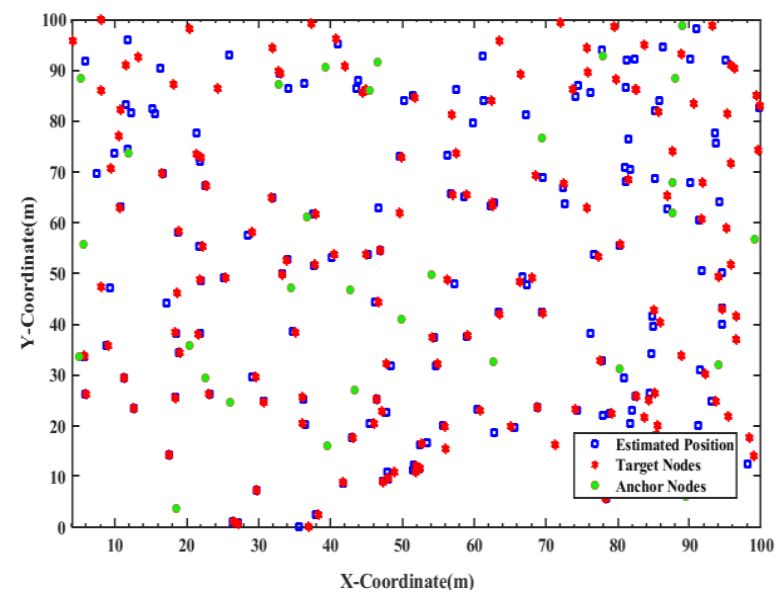

Fig.8. Localization of nodes using EHO 


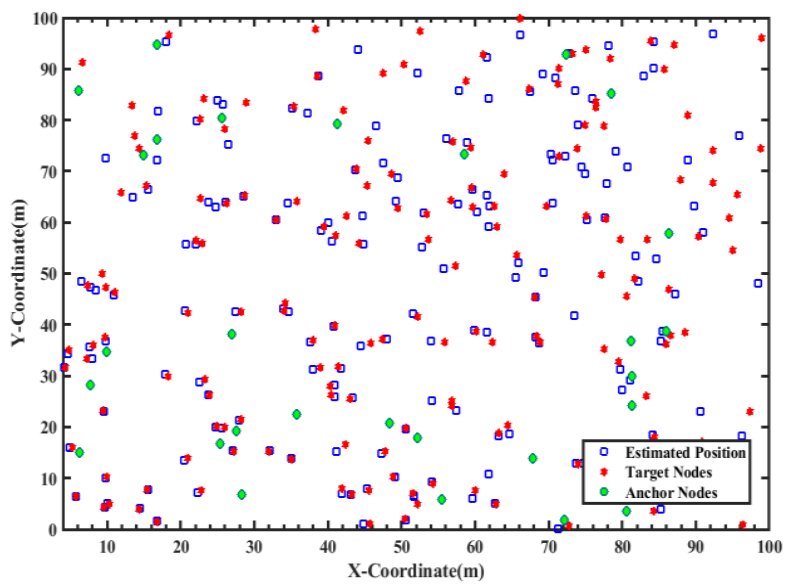

Fig.9. Localization of nodes using DV-Hop

\section{Simulation Results and Discussions}

\subsection{Analysis of varying anchor node on localized nodes}

The figure 10 denoted that the number of localized nodes is higher by the LOSSADE model whereas the DV-Hop model has attained minimum number of localized nodes. At the same time, the SSA, BSA, GWO, and EHO algorithms have exposed rather better and less accurate localization performance by obtaining sparse number of localized nodes. Although GWO algorithm has attained more localized nodes compared to DV-Hop models, it failed to outperform the proposed method. For instance, under the anchor count of 10, the DV-Hop model has attained minimal number of 82 localized nodes. At the same time, the SSA, EHO, GWO and BSA models have attained a moderate number of localized nodes such as 112, 99,108, and 104 respectively. However, the proposed method has properly localized a maximum of 130 nodes. Similarly, in the presence of 50 anchor nodes, the DV-Hop methods have reached a lower number of 107 localized nodes. In line with this, GWO outperformed the DV-Hop models by obtaining 128 localized nodes. Simultaneously, the SSA, GWO, EHO, and BSA have reached a considerable number of localized nodes like 167, 153, 128 and 144 correspondingly. Hence, the presented LOSSADE properly localized a higher value of 189 nodes.

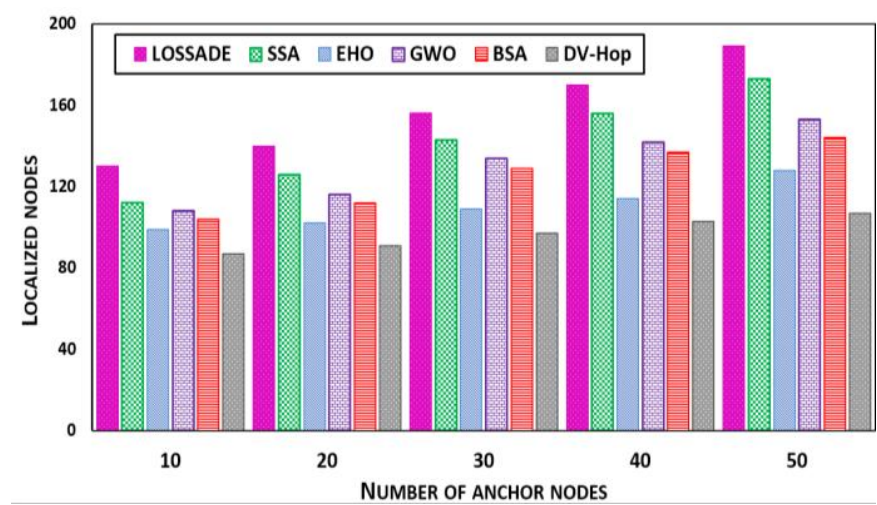

Fig.10. Analysis of number of localized nodes versus known nodes using LOSSADE model

\subsection{Analysis of localization rate (LR) by varying number of anchor nodes}

Localization rate is the success percentage defined as the ratio of the localized node to a total number of target nodes in the network. It measures the quality of the localization algorithm as in equation (21). Higher the localization rate refers that a greater number of nodes position are found. If low localization rate, then the nodes localized are of a smaller number.

$$
\text { Localization Rate }=\frac{\text { Number of localized nodes }}{\text { Number of unlocalized nodes }}
$$

Figure 11 shows the localization performance of the LOSSADE model in terms of localization rate under a varying number of anchor nodes. The figure denoted that the localization rate is higher by the LOSSADE model while the DVHop model has attained only a lower localization rate. At the same time, the SSA, EHO, GWO, and BSA algorithms have shown better and closer localization performance by obtaining a high localization rate. Although the GWO 
algorithm has attained more localized nodes compared to the DV-Hop models, it failed to outperform the proposed method. For instance, under the anchor count of 10, the DV-Hop models have attained a localization rate of 0.44 . Along with that, the GWO algorithm has surpassed the DV-Hop model by attaining a localization rate of 0.50 . At the same time, the SSA, EHO, GWO, and BSA models have obtained moderate localization rates such as $0.56,0.50,0.54$, and 0.52 correspondingly. Still, the proposed technique has reached a higher localization rate of 0.62 . Along with that, under the existence of 50 anchor nodes, the DV-Hop model has accomplished a localization rate of 0.54 . In line with this, the EHO model has surpassed the DV-Hop by reaching a localization rate of 0.64. Meantime, the SSA, EHO, GWO, and BSA approaches have achieved a gradual localization rate like $0.84,0.77,0.68$, and 0.72 respectively. Thus, the presented LOSSADE method has attained a maximum localization rate of 0.92 .

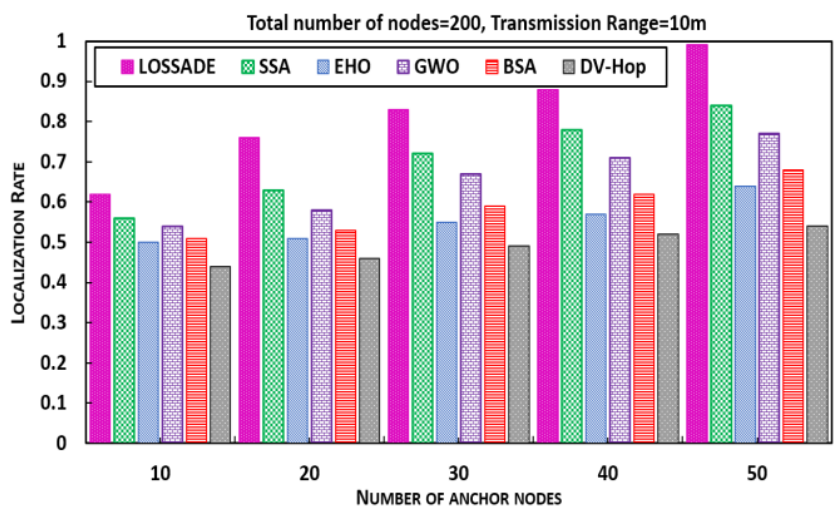

Fig.11. Analysis of number of anchor nodes versus localization rate using LOSSADE model

\subsection{Analysis of varying number of anchor nodes versus the convergence rate $(C R)$ for cost}

The convergence rate is the time required for the localization of each node about the localization rate. The time gets affected by the network area. This is given in equation (22)

$$
\text { Convergence Rate }=\frac{\text { Time of localization }}{\text { Localization rate }}
$$

Figure 12 depicts the localization cost of the LOSSADE model to convergence rate under varying counts of anchor nodes. The convergence rate of the proposed model shows that the complexity is constructed on SSA algorithms based on the basic Doppler effect in terms of localization time. The figure implied that the convergence rate is lower by the LOSSADE model while the DV-Hop approach has reached a maximum convergence rate. Similarly, the SSA, GWO, EHO, and BSA methodologies have depicted moderate and closer localization performance by accomplishing a better convergence rate. Even though the GWO as achieved a better convergence rate than the DV-Hop model, it failed to perform well than the projected technique. Under the anchor count of 10, the DV-Hop models have reached a greater convergence rate of 76. In line with this, the BSA algorithm has surpassed the DV-Hop approach by attaining a convergence rate of 74. Simultaneously, SSA, GWO, EHO, and BSA methodologies have carried out gradual convergence rates such as $45,65,71$, and 74 correspondingly. Therefore, the projected approach has reached a lower convergence rate of 41. Likewise, in the existence of 50 anchor nodes, the DV-Hop models have reached a convergence rate of 63.In the same way, the BSA algorithm has performed the DV-Hop by accomplishing a convergence rate of 61. Meantime, the SSA, GWO, and EHO have reached gradually convergence rate like 22, 41, and 54 correspondingly. Hence, the presented approach has reached lower convergence rate of 15 .

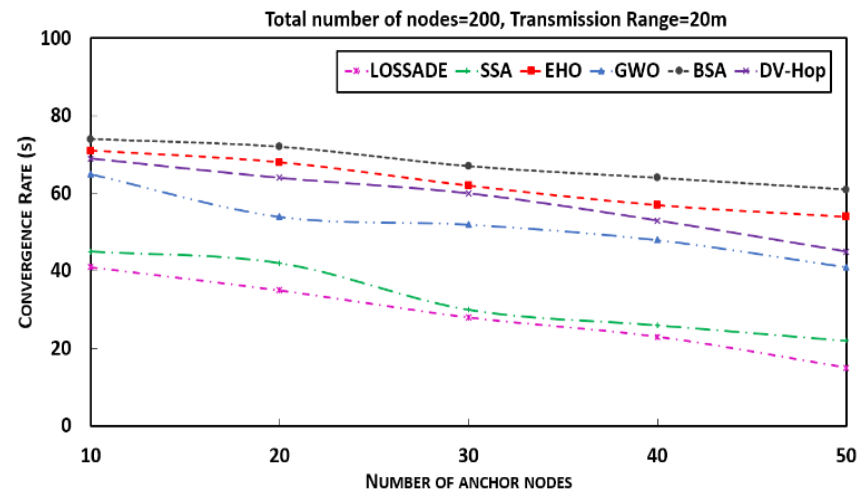

Fig.12. Analysis of number of known nodes versus convergence rate using LOSSADE model 


\subsection{Analysis of Localization Error (LE) with varying number of anchor nodes}

As in equation (23-26), the localization error is the Mean Square Error (MSE) amid the estimated and the real location of the target nodes in the given network size. Where ' $\mathrm{NL}$ ' is the number of target nodes for $\mathrm{i}=1,2 \ldots \mathrm{N}$ node, determined node coordinates denoted $\left(X_{i}, Y_{i}\right)$ and the actual node coordinates represented as $\left(x_{i}, y_{i}\right)$.

$$
\begin{aligned}
& \text { Localization Error }(\mathrm{LE})=\sqrt{\left(\mathrm{x}_{\mathrm{i}}-\mathrm{X}_{\mathrm{i}}\right)^{2}+\left(\mathrm{y}_{\mathrm{i}}-\mathrm{Y}_{\mathrm{i}}\right)^{2}} \\
& \text { Average } L E=\frac{1}{N L} \sum_{i=1}^{N} \sqrt{\left(x_{i}-X_{i}\right)^{2}+\left(y_{i}-Y_{i}\right)^{2}} \\
& R M S E=\sqrt{\frac{1}{N L} \sum_{i=1}^{N}\left(x_{i}-X_{i}\right)^{2}+\left(y_{i}-Y_{i}\right)^{2}} \\
& \text { Localization accuracy }=\frac{\sum_{i=1}^{n} \sqrt{\left(\mathrm{x}_{\mathrm{i}}-\mathrm{X}_{\mathrm{i}}\right)^{2}+\left(\mathrm{y}_{\mathrm{i}}-\mathrm{Y}_{\mathrm{i}}\right)^{2}}}{n \times R^{2}}
\end{aligned}
$$

Figure 13 displays localization error under different anchor node count. The bar chart portrays that the localization error is lower by the LOSSADE model while the DV-Hop model has reached a maximum localization error. Simultaneously, the SSA, EHO, GWO, and BSA has moderate localization error. Even though the GWO model has obtained better localization error compared to the DV-Hop model, it failed to perform the presented model. For the sample, under the existence of 10 anchor nodes, the DV-Hop methodologies have accomplished maximum localization error of 1.45. Similarly, GWO has outperformed DV-Hop technique by reaching a localization error of 0.53 . Concurrently, the SSA, EHO, GWO, and BSA approaches have reached better localization error $0.45,0.74,0.53$, and 0.72 respectively. However, the proposed method has attained minimum localization error of 0.38 . Similarly, in the presence of 50 anchor nodes, the DV-HOP model has attained localization error of 0.97 . Along with that, the EHO algorithm has surpassed the DV-HOP model by attaining a localization error of 0.74 . At the same time, the SSA, GWO, and BSA models have attained moderate localization errors such as $0.32,0.38$, and 0.57 respectively. However, the proposed LOSSADE method has attained minimum localization error of 0.18 .

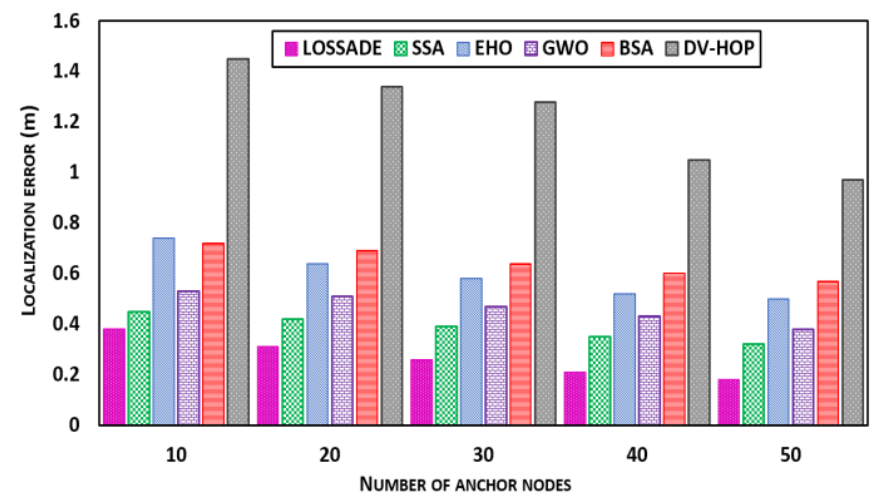

Fig.13. Analysis of localization error with respect to transmission range in LOSSADE model

\subsection{Analysis of localization error (LE) for accuracy}

Figure 14 demonstrates the localization performance of the LOSSADE with respect to localization error under diverse transmission range. The figure implied that the localization error is lower by the proposed approach while the DV-Hop model has accomplished a maximum localization error. Meantime, SSA, GWO, EHO, and BSA shows better and identical localization performance by attaining moderate localization error. Even though the BSA has reached better localization error than DV-Hop model, it failed to perform better the proposed method. For instance, under transmission range of $10 \mathrm{~m}$, the DV-Hop approach has reached maximum localization error of 0.97 . In line with this, the BSA model has surpassed the DV-Hop by reaching a localization error of 0.53. Simultaneously, the SSA, GWO, EHO, and BSA approaches have reached considerable localization errors like $0.32,0.5,0.38$, and 0.53 respectively. Hence, the presented approach has attained a lower localization error of 0.18 . In line with this, in the presence of a communication range of 50m, DV-Hop frameworks have reached localization errors of 0.83 . In same way, the BSA algorithm has performed well than the DV-Hop models by obtaining a localization error of 0.41 . Meantime, SSA, GWO, EHO, and BSA have reached gradual localization errors $0.13,0.35,0.24$, and 0.41 respectively. Therefore, the presented method has reached least localization error of 0.07 . 


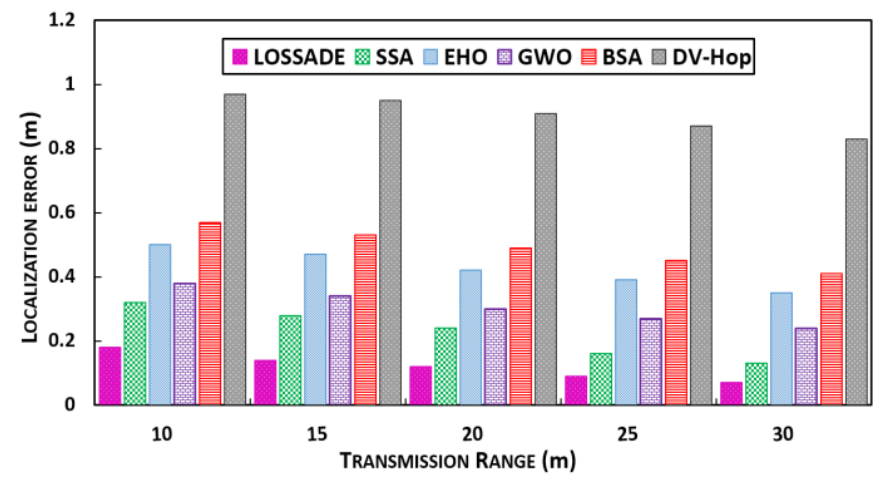

Fig.14. Analysis of localization error with respect to transmission range in LOSSADE model

\subsection{Analysis of localization error (LE) with varying ranging error}

Figure 15 unravels the localization error with the ranging error of 5-25\% of the LOSSADE. The figure depicted that the localization error is lower by the LOSSADE model while the DV-HOP has accomplished a maximum localization error. Meantime, the SSA, GWO, EHO, and BSA models have showcased moderate and identical localization performance by attaining considerable localization error. Even though the BSA technology has reached better localization error related to the DV-HOP methodology, it failed to perform well with the projected approach.

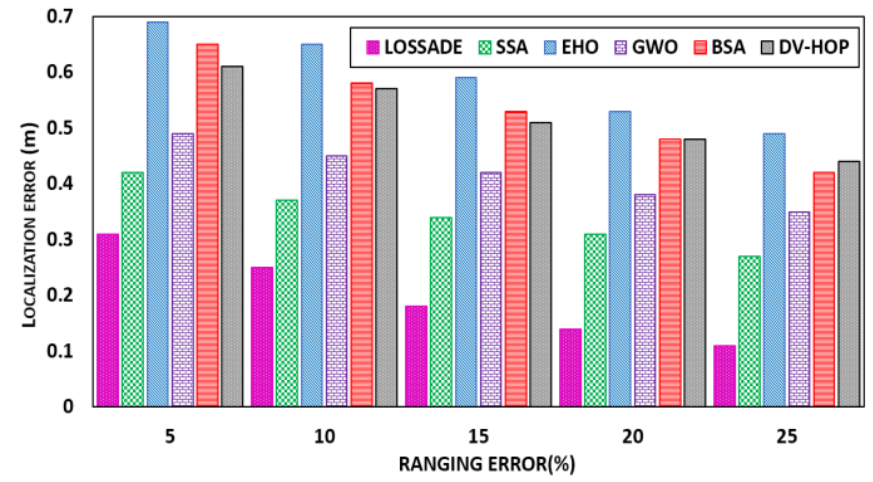

Fig.15. Analysis of localization error with respect to Ranging error in LOSSADE model

\section{Conclusion}

In this paper we propose a hybrid node localization model that collaborates the metaheuristic algorithm named Salp Swarm optimization Algorithm with Doppler Effect (LOSSADE) for effective node positioning to increase the performance of the network. The LOSSADE model incorporates the Doppler Effect effectively to localize maximum number of unknown nodes by considering Euclidian distance between the nodes. The salp periodically moves its body producing the current waves that travel out words in all direction at same speed since it is in the same water medium. The leader salp moves in random manner and maximizes the frequency. The location of the salp leader and the prey will get updated using the Doppler shift. The unknown node location is calculated by the trilateration method with help of salp swarm algorithm fitness function. The presented localization model uses several metrics like localization error, discovered nodes, localization rate, and localization time under a varying number of anchor nodes, ranging error, and transmission range to achieve low localization error in less computation time and to maximize the network lifetime. The performance of the LOSSADE model has been verified using MATLAB and the obtained results indicated that the proposed algorithm has attained maximum localization and convergence rate related to other methods. For the future work, the performance of the localization process such as energy consumption can be minimized with a novel hybrid approach using either metaheuristic or heuristic algorithms. This can also be applied to further improve the node localization on real time field like Internet of Things (IoT), and in three dimensional (3D) WSN scenarios

\section{Declaration of Competing Interest}

The authors declare that they have no known competing financial interests or personal relationships that could have appeared to influence the work reported in this paper. 


\section{References}

[1] Han, G., Xu, H., Duong, T.Q., Jiang, J., Hara T, "Localization algorithms of wireless sensor networks: A survey", Springer journal of telecommunication systems, Volume 52, Issue 4, 2013, Pages 2419-2436

[2] D.Niculescu, B.Nath, "DV based positioning in ad-hoc networks," Telecommunication Systems, Volume 22,Issue 1-4,Pages. 267-280, 2003.

[3] Ahmed k. Daraj, Alhamzah T. Mohammad, Mahmood F. Mosle, "Indoor Localization Enhancement Based on Time of Arrival Using Sectoring Method", International Journal of Intelligent Systems and Applications (IJISA), Vol.12, No.3, Jun. 2020, PP.17

[4] M. B. Nirmala, A. S. Manjunatha, "Enhanced Voting based Secure Localization for Wireless Sensor Networks ", International Journal of Computer Network and Information Security (IJCNIS), Vol.7, No.12, Nov. 2015, PP.52-59

[5] Santar Pal Singh, S. C. Sharma," Critical Analysis of Distributed Localization Algorithms for Wireless Sensor Networks“, International Journal of Wireless and Microwave Technologies(IJWMT), Vol.6, No.4, Jul. 2016, PP.72-83

[6] D.H.Wolpert, W.G.Macready ,"No-free- lunch theorems for optimization "IEEE transactions on evolutionary computation, Volume 1, Issue 1, 1997, Pages 67-82

[7] Miloud Mihoubi, Abdellatif rahmoun, Pascal Lorenz, Noureddine Lasla, "An effective bat algorithm for node localization in distributed wireless sensor network," Security and privacy, Volume 1, Issue 1, Pages 1-20, 2018.

[8] Strumberger, I.; Beko, M.; Tuba, M.; Minovic, M.; Bacanin, N. Elephant Herding Optimization Algorithm for Wireless Sensor Network Localization Problem," In Technological Innovation for Resilient., Springer International, 2018; Pages 175-184.

[9] Wang, G.G.; Deb, S.; Gao, X.Z.; Dos Santos Coelho, L. A new metaheuristic optimization algorithm motivated by elephant herding behavior. International Journal of Bio-Inspiration Computing, 2017, 8, Pages 394-409.

[10] Sankalap Arora, Satvir Singh, "Node Localization in Wireless Sensor Networks Using Butterfly Optimization Algorithm", springer journal of science and engineering, Volume 42, 2017, Pages 3325-3335

[11] Amrita Datta, Mou Dasgupta, "On accurate localization of nodes in underwater sensor networks: a Doppler shift and modified genetic algorithm-based localization technique," springer journal of Evolutionary Intelligence, 2019.

[12] Kussat NH, Chadwell CD, Zimmerman R, "Absolute positioning of an autonomous underwater vehicle using GPS and acoustic measurements", IEEE journal of Ocean Engineering, Volume 30, Issue 1, Pages153-164, 2005.

[13] Yan J, Zhang X, Luo X, Wang Y, Chen C, Guan X, "Asynchronous localization with mobility prediction for underwater acoustic sensor networks", IEEE journal of Transactions on Vehicular Technology, volume 67, Issue 3, Pages 2543-2556, 2017.

[14] Han Y, Zhang J, Sun D, "Error control and adjustment method for underwater wireless sensor network localization”, Elsevier journal of Applied Acoustics, Volume 130, Pages 293-299, 2018.

[15] Nagireddy V, Parwekar P, Mishra TK, "Velocity adaptation based PSO for localization in wireless sensor networks", springer journal of Evolutionary Intelligence, 2018

[16] Phoemphon, S., So-In, C., Leelathakul, N, "A hybrid localization model using node segmentation and improved particle swarm optimization with obstacle awareness for wireless sensor networks", Springer journal of expert systems with applications, Volume 143, 2020.

[17] Huthaifa M. Kanoosh,Mazen M. Selim,Essam Halim Houssein, "Salp Swarm Algorithm for Node Localization in Wireless Sensor Networks", ACM Journal of Computer Networks and Communications, 2019.

[18] Zhou, G., He, T., Krishnamurthy, S., Stankovic, J, "Models and solutions for radio irregularity in wireless sensor networks", ACM Transactions on Sensor Networks, Volume 2, Issue 2, Pages 221-262, 2006.

[19] S. Mirjalili, S. M. Mirjalili and A. Lewis, Grey wolf optimizer: Theory, Literature Review, and Application in Computational Fluid Dynamics Problems, Advances in Engineering Software, Volume 69, Pages 46-61, 2014.

\section{Authors' Profiles}

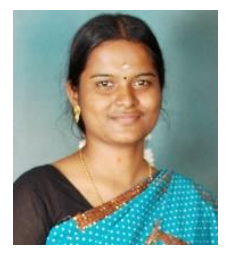

Panimalar Kathiroli has completed M. Tech Degree-2011 and B. Tech Degree-2009 in the department of Computer Science and Engineering from Sri Manakula Vinayagar Engineering College, Puducherry. Since 2011, She has worked as Assistant professor in the Department of Computer Science and Engineering in various engineering colleges. Currently she is pursuing as a research scholar in the department of Computer Science and Engineering, Pondicherry Engineering college. She has Published and presented about 20 papers in National/International journals and conferences. She is a lifetime member of ISTE and IAENG.

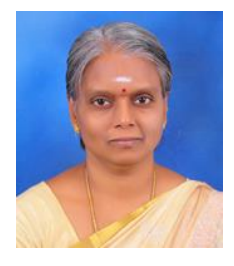

S. Kanmani obtained her B.E Degree (University Third Rank)-1990 and M.E Degree (University first Rank)1991 in department of Computer Science and Engineering from Government College of Technology, Coimbatore. She has completed her Ph. D-2006 in college of engineering, Guindy Anna University, Chennai in software measurement and testing. She has served as a lecturer in Annamalai University and in Pondicherry engineering college in the Department of Computer Science and Engineering. Since then she was in various positions in the Pondicherry engineering college and now, she is a professor in the Department of Information Technology. She has published around 250 papers in the international conferences and reputed journals. She is a reviewer for many 
international journal publications. Her areas of interest are meta heuristic algorithms, intelligent computing, and software testing. She is a member of computer society of India, ISTE and institute of engineers India.

How to cite this paper: Panimalar Kathiroli, Kanmani. S, "Localization by Salp Swarm Optimization with Doppler Effect in Wireless Sensor Networks", International Journal of Computer Network and Information Security(IJCNIS), Vol.13, No.6, pp.26-40, 2021. DOI: 10.5815/ijcnis.2021.06.03 